\title{
Bilateral recurrent haemorrhagic pleural effusion in asymptomatic chronic pancreatitis
}

\author{
S C TEWARI, R JAYASWAL, M S CHAUHAN, S K KAUL, V A NARAYANAN \\ From the Department of Tuberculosis and Chest Diseases, Cardiothoracic Centre, Military Hospital, and the \\ Department of Medicine, Armed Forces Medical College, Pune, India
}

ABSTRACT In a case of bilateral recurrent haemorrhagic pleural effusion due to asymptomatic pancreatitis the diagnosis was suggested by the presence of amylase in the pleural fluid when other possible causes had been excluded. Abdominal computed tomography and laparotomy confirmed the diagnosis. No communications could be seen between the peritoneal and pleural space at laparotomy.

Pleural effusion is an uncommon complication of pancreatitis. It is often left sided and associated with acute pancreatitis. ${ }^{12}$ Development of massive and recurrent haemorrhagic pleural effusion on one side followed by effusion on the opposite side after a relatively symptom free interval in a patient with no clinical evidence of pancreatic disease must be very rare. ${ }^{3}$

\section{Case report}

A 38 year old man, a hospital store keeper in the army, presented with cough and dyspnoea of seven days' duration on accustomed exertion. The findings from clinical and radiological examination were consistent with a massive left sided pleural effusion (figure). The patient denied any past illness except for a vague abdominal pain nine months earlier, which had lasted for 12 hours. Pleural fluid was port wine colour and contained $72 \mathrm{~g} / 1$ protein, $3.3 \mathrm{mmol} / 1$ glucose, (blood glucose $5.4 \mathrm{mmol} / \mathrm{l}$ ), cells $0.4 \times 10^{9} / 1$ (mostly lymphocytes), red blood cells $10.8 \times 10^{9} / 1$. No malignant cells or acid fast organisms were seen on smears and cultures were sterile. Bronchoscopy and bronchoalveolar lavage studies were noncontributory. Pleural biopsy showed normal pleura.

Malignant causes having been reasonably excluded and because tuberculosis is an extremely common cause of pleural effusion in India, the patient was treated with a short term chemotherapeutic regimen of isoniazid, rifampicin, pyrazinamide, and streptomycin for two months followed by isoniazid and rifampicin daily for seven months. Prednisolone $1 \mathrm{mg} / \mathrm{kg}$ was given initially with reduction of the dose within four weeks.

Address for reprint requests: Lt Col S C Tewari, Department of Respiratory Medicine, Military Hospital (CTC), Pune-41 1040, India.
The pleural effusion continued to accumulate and 4.5 litres of fluid were aspirated on four occasions over the next threeo weeks. Thoracic computed tomography showed no pul $\rightarrow$ monary, pleural, or mediastinal lesion except for the pleurap effusion. As the patient was becoming dyspnoeic a left thoracotomy was done; this showed slight thickening of the parietal pleura over the upper lobe. A pleura biopsy speciment showed no specific abnormality. An abrasive pleurodesis was carried out and he remained symptom free for the next $12 \mathbb{D}$ weeks.

A similar massive haemorrhagic pleural effusion was thero found on the right side (figure). Three litres of fluid were removed in four sessions. As all common causes had been reasonably excluded the amylase activity of the pleural fluid was measured and found to be very high at 48900 Somogy? units/l. Blood amylase activity was normal (120 Somogyn $\mathrm{U} / 1)$. Computed tomography of the abdomen showed an enlarged head of the pancreas containing cystic lesions; no calcification was seen. Abdominal ultrasound did not show $\vec{b}$ gallstones. As the pleural fluid continued to collect despite aspirations a laparotomy was carried out. This showed gross dilatation of the pancreatic duct with periductal fibrosis. The? diaphragm was inspected and showed no congenital defects There was no fluid in the lesser sac and no othe pleuroperitoneal communication could be found. A panஹِ creaticojejunostomy was carried out.

On the ninth postoperative day the patient suddenly died as a result of shock, which necropsy showed to be due to digestion of a medium sized artery at the coeliac axis with massive intra-abdominal haemorrhage.

\section{Discussion}

Pleural effusion is well recognised in acute pancreatitis, when the diagnosis is usually obvious. Ascites or lesser sac fluido collection in chronic pancreatic disease may also be associated with pleural effusion. Pancreatic disease is generally not considered in patients presenting solely withw massive haemorrhagic pleural effusion, particularly when this is bilateral and recurrent. Possible mechanisms pose tulated in producing pleural effusion in acute pancreatic disease are direct contact of pancreatic enzymes with thes diaphragm and consequent diaphragmatic pleural inflamma tion, haematogenous or lymphogenous transdiaphragmatic carriage of enzymes to the pleura, and direct movement of fluid from the abdomen to the thorax. ${ }^{45}$ This last mechanism? is most likely to operate in chronic pancreatitis.

The diagnosis was not suspected initially in our case as the् 


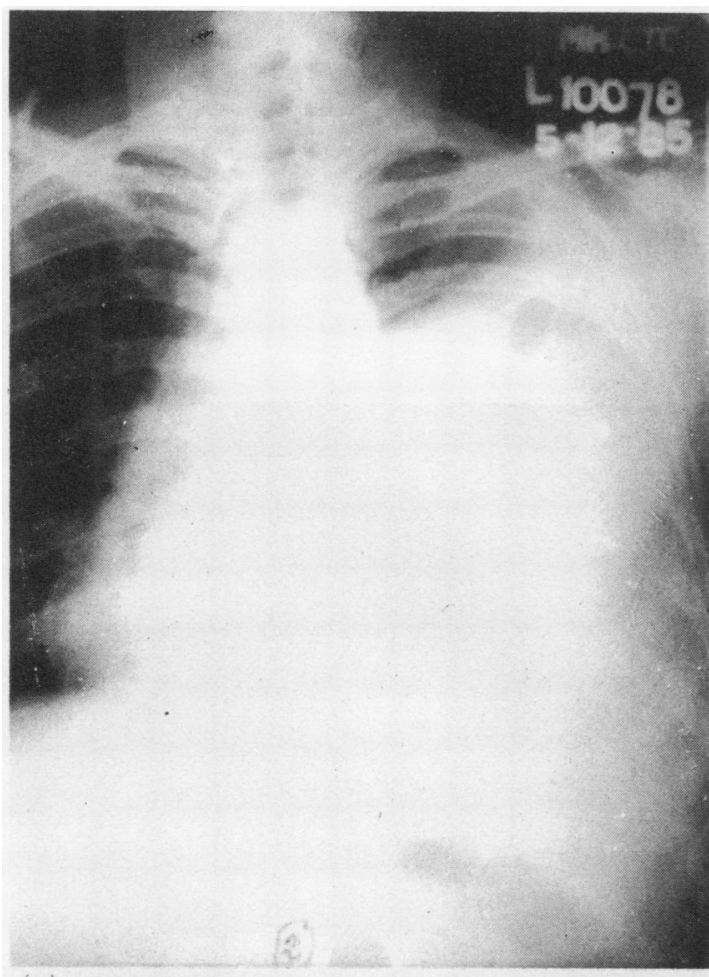

(a)

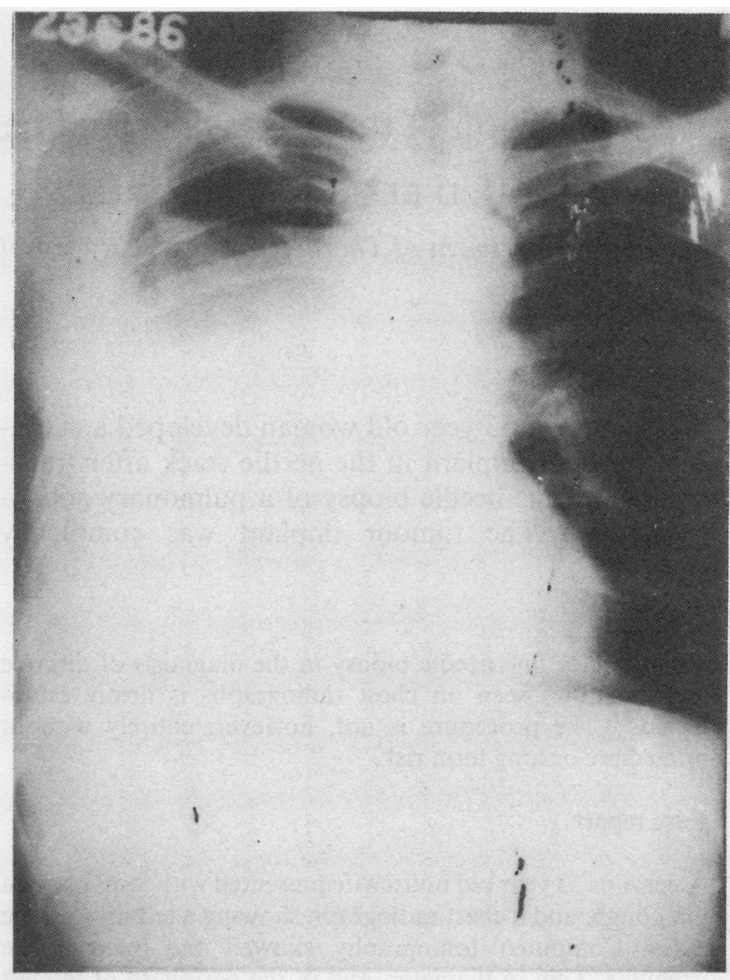

(b)

Posteroanterior chest radiographs showing (a) left sided and (b) right sided pleural effusion.

patient did not have symptoms and signs to suggest pancreatic disease. He did not drink alcohol and had no symptoms to suggest gall bladder or pancreatic disease in the past. The diagnosis was suggested by the very high amylase content of the pleural fluid and confirmed by computed tomography.

Massive bilateral haemorrhagic pleural effusion with a high pleural fluid amylase was reported by Miridjanian et al. ${ }^{3}$ Some degree of pleural thickening, as in our case, has been reported by earlier workers ${ }^{6}$; but no specific changes have been noted on biopsy specimens.

This case highlights the value of measuring the amylase activity in pleural fluid of obscure cause and the value of computed tomography in confirming the diagnosis.' It is also of interest that no communication was seen between peritoneal and pleural spaces to explain the occurrence of these massive and recurrent bilateral effusions.

\section{References}

1 Kaye MD. Pleuropulmonary complications of pancreatitis. Thorax 1968;23:297-306.

2 Light RW, Ball WC. Glucose and amylase in pleural effusions. JAMA 1973:225:257-60.

3 Miridjanian A, Ambruoso VN, Derby BM, Tice DA. Massive bilateral haemorrhagic pleural effusions in chronic relapsing pancreatitis. Arch Surg 1969;98:62-6.

4 Anderson WJ, Skinner DB, Zuidema GD, Cameron JL. Chronic pancreatic pleural effusions. Surg Gynaecol Obster 1973;137:827-30.

5 Tombroff M, Loicq A, de Koster J-P, Engleholm L, Govaerts J-P. Pleural effusions with pancreaticopleural fistula. Br Med J 1973; i:330-1.

6 Shapiro DH, Anagnostopoulos CE, Dineen JP. Decortication and pleurectomy for the pleuropulmonary complications of pancreatitis. Ann Thorac Surg 1970;9:76-80.

7 Spiro HM. Clinico gastroenterology. 3rd ed. New York: MacMillan, 1983:1194-233. 